\title{
Adaptive predictors based on probabilistic SVM for real time disruption mitigation on JET
}

\section{Murari, A.}

2018

Murari , A , JET Contributors \& Ahlgren , T 2018 , ' Adaptive predictors based on probabilistic SVM for real time disruption mitigation on JET ' , Nuclear Fusion , vol. 58 , no. 5 , 056002 . https://doi.org/10.1088/1741-4326/aaaf9c

http://hdl.handle.net/10138/326705

https://doi.org/10.1088/1741-4326/aaaf9c

unspecified

acceptedVersion

Downloaded from Helda, University of Helsinki institutional repository.

This is an electronic reprint of the original article.

This reprint may differ from the original in pagination and typographic detail.

Please cite the original version. 


\title{
Adaptive Predictors based on Probabilistic SVM for Disruption Mitigation, Avoidance and Classification on JET
}

EUROfusion Consortium, JET, Culham Science Centre, Abingdon, OX14 3DB, UK

\author{
by A.Murari ${ }^{1}$, M.Lungaroni ${ }^{2}$, E.Peluso ${ }^{2}$, P.Gaudio $^{2}$, J.Vega $^{3}$, S.Dormido-Canto ${ }^{4}$, M.Baruzzo $^{1}$ \\ and M.Gelfusa ${ }^{2}$ and JET Contributors* \\ 1) Consorzio RFX (CNR, ENEA, INFN, Universita' di Padova, Acciaierie Venete SpA), Corso Stati Uniti 4, \\ 35127 Padova, Italy. \\ 2) Department of Industrial Engineering, University of Rome "Tor Vergata", via del Politecnico 1, \\ Roma, Italy \\ 3) Laboratorio Nacional de Fusión, CIEMAT. Av. Complutense 40. 28040 Madrid. Spain \\ 4) Dpto. de Informática y Automática UNED Juan del Rosal 16. 28040 Madrid. Spain
}

\begin{abstract}
*See the author list of "Overview of the JET results in support to ITER" by X. Litaudon et al. to be published in Nuclear Fusion Special issue: overview and summary reports from the 26th Fusion Energy Conference (Kyoto, Japan, 17-22 October 2016)
\end{abstract}

\begin{abstract}
Detecting disruptions with sufficient anticipation time is essential to undertake any form of remedial strategy, mitigation or avoidance. Traditional predictors based on machine learning techniques can be very performing if properly optimised but do not provide a natural estimate of the quality of their outputs. In this paper a new set of tools, based on probabilistic extensions of Support Vector Machines, are introduced and applied for the first time to JET data. The probabilistic output constitutes a natural qualification of the prediction quality and provides additional flexibility. Indeed the versatility of the developed techniques is such that different versions of the tools can be optimised to perform various tasks, from prediction for mitigation to avoidance or even classification. Large databases of disruptions, covering entire campaigns, are analysed, both for the case of the graphite and the ITER Like Wall. Success rates of the order of $97 \%$ with about $4.5 \%$ of false alarms can be easily achieved, satisfying even the requirements of the next generation of devices. The fact that the developed tools give the probability of disruption improves the interpretability of the results, provides an estimate of the predictor quality and gives new insights into the physics. Moreover, a probabilistic treatment permits to insert more easily these classifiers into general decision support and control systems.
\end{abstract}

Keywords: Disruptions, Probabilistic SVM, Machine Learning Predictors, Decision Support Systems

Corresponding author: emmanuele.peluso@uniroma2.it 


\section{Machine learning approaches to disruption prediction in Tokamaks and probabilistic SVM}

In the last years, collapses and their causes have become not only a major field of research but have also captured the attention of the mainstream media. From market crashes to earthquakes and structural failures in civil engineering, increasing attention is devoted to surprising and typically unexpected abrupt changes in systems, leading to catastrophic consequences. The statistical investigation of these phenomena, particularly for robust prediction, requires the development of new mathematical tools [1]. The systematic use of machine learning methods for this purpose is continuously increasing.

In Tokamaks, disruptions remain the most serious cause of collapse, leading to the abrupt quenching of the plasma with potential major risks for the structural integrity of the devices. The percent of disruptions allowed in ITER is quite limited [2]. But disruptions are also a serious issue for the present largest devices. For example, they are one of the main impediments to systematic high current operation in JET, particularly now that the new combination of materials, $\mathrm{Be}$ in the main chamber and $\mathrm{W}$ in the divertor, renders the first wall less forgiving than in the past. Moreover, high triangularity plasma scenarios are also strongly penalised by the high probability and high forces of their disruptions.

Given their potential impact on the integrity of the devices, disruptions are a subject of extensive research at present. Various methods of mitigation are being investigated, particularly massive gas injection [2]. The main objective of these techniques consists of limiting the energy conducted directly to the wall by converting the highest percentage of it into radiation. On the other hand, these conversion methods have not only to be effective but also are required not to pose themselves other hazards to the machines, such as excessive increases of the eddy currents due to very fast current quenches. To reduce the strain on the devices also avoidance tactics are being considered, to undertake remedial actions and prevent the occurrence of disruptions. This is particularly important in the perspective of DEMO, since in the demonstrative fusion reactor unmitigated disruptions will have to be almost completely avoided and the number of mitigated ones minimised [2].

Of course, reliable prediction tools are a prerequisite to any mitigation or avoidance strategy. Unfortunately, the theoretical understanding of the causes of disruptions is not sufficient to guarantee reliable predictions. As a consequence, prediction models based on first principles are virtually non-existent. Therefore, in the last decades, a lot of efforts have been devoted to developing empirical models, capable of launching an alarm when a disruption is approaching. These empirical models are either manual or based on machine 
learning tools. On JET the main manually adjusted tool is the Locked Mode Predictor based on a Threshold criterion (LMPT), which triggers mitigation actions when the signal of the locked mode amplitude reaches a certain threshold. This solution results in so called "ephemeral predictors", i.e. systems which age very quickly and require frequent adjustments to remain effective. Indeed, in the case of LMPT, the threshold has to be adjusted quite frequently and certainly more than once per experimental campaign, not always at an optimal level [3].

Nowadays machine learning tools are deployed in a variety of domains to support, and even to perform, the entire decision making process [4]. Following this general trend, various generations of predictors based on machine learning tools have also been applied to JET data in the last decades. Various alternatives have been explored, ranging from Neural Networks to Self Organizing Maps and fuzzy decision trees [5-8]. Two of the most performing, APODIS and SPAD, have been deployed in JET real time network. APODIS, which has even been used in closed loop for triggering mitigation actions, is a typical data mining tool, using a complex Support Vector Machines (SVM) classifier and requiring a large training set [9]. SPAD implements an anomaly detection strategy [10-12] and does not need a large number of disruptions for learning a model. Some adaptive prediction tools have also been developed and have been applied off line to entire campaigns $[13,14]$. These predictors are quite complex and in general, apart from [13], do not provide as output a probability, a fact which can render their interpretation and integration into general control systems quite involved.

In this paper, an adaptive predictor, which provides a probabilistic output, is described in detail. The predictor is based on an SVM classifier, whose estimates are processed with a Bayesian treatment to provide the probability that a discharge is in a disruptive state. A "learning from scratch" approach has been implemented for the training and therefore the predictor can start working with just one disruptive and one non disruptive example $[13,14]$. The model obtained with the first examples is then modified as the campaign progresses, by refining the training with additional cases. With an appropriate choice of the prior (see Section 2), the probability of disruption provided by the classifier allows optimising the various remedial strategies, from mitigation to avoidance. Moreover, the approach is fully compatible with a more targeted strategy, aimed at optimising the predictors for specific types of disruptions; this can be achieved by an appropriate selection of the training set and probability thresholds. Specific versions of the predictors have also been developed, to determine the disruption type. The proposed techniques therefore constitute a multipurpose 
methodology capable of providing a wide set of tools to cover all the main tasks involved in disruption forecasting for Tokamaks: prediction for mitigation, prediction for avoidance and disruption type classification. Predictors of this family are therefore referred to as MAPP (Multipurpose Adaptive Probabilistic Predictors). It is worth emphasizing the fact that, in addition to providing very competitive performance compared to other machine learning methods, the proposed tools present the unquestionable advantage of expressing the output in terms of classic probabilities. This aspect no only renders the tools easier to interpret by the user but they can also provide better insight in the underlying phenomenology. Moreover probabilities constitute a quite natural and common method to qualify the confidence in the prediction. Therefore, the probabilistic output is fully suited to the integration of the proposed classifiers into more general decision support systems. In particular MAPP can easily become part of more complex logics of intervention for both mitigation and avoidance.

Regarding the structure of the paper, next section gives an overview of the mathematical background required for a basic understanding of traditional SVM and their probabilistic extensions. Section 3 discusses in detail the adaptive method adopted to train the various versions of MAPP and describes the main characteristics of JET databases investigated. The results obtained for the ILW are reviewed in Section 4 and those for the JET with a carbon wall in Section 5; more details are given in the Appendixes. The potential of probabilistic SVM for avoidance and disruption classification is discussed in Section 6 and 7

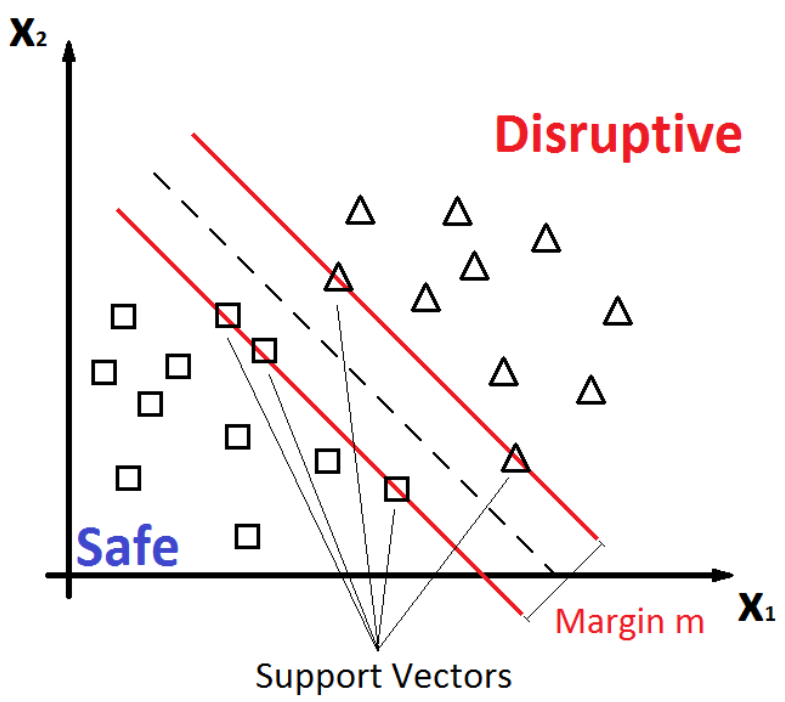

Figure 1. SVM classification: the success rate is optimised by a linear hyperplane that maximizes the separating margin between the two regions, disruptive and non-disruptive, in feature space. respectively. The conclusions and lines of future work are the subject of the last Section 8 of the paper. 


\section{Probabilistic SVM for Classification: the mathematical background}

Machine learning techniques have become quite common to attack problems so complex that cannot be solved with more traditional algorithmic methods. They are typically deployed to extract useful information from large amount of multidimensional data. Machine learning tools can perform many different tasks, including classification, regression and anomaly detection. In the arsenal of machine learning methods, SVMs are very powerful and present various very desirable properties for scientific applications, such as determinism (of the training process) and structural stability [15]. They are used as various types of classifiers for the studies described in this paper. In this section, the background on the traditional SVM and their probabilistic versions are briefly overviewed. The aim is not to give a full treatment of SVMs, which can be found in the references, but to provide a concise treatment to allow the reader to appreciate the probabilistic extensions, which are more recent developments not completely covered in the non-specialist literature.

\subsection{The basics of SVM}

Binary classification is a classic application of machine learning. Given a set of examples, which belong to two different classes, the SVM proceeds by projecting these inputs into a high-dimensional space through some suitable non-linear mapping. In this high

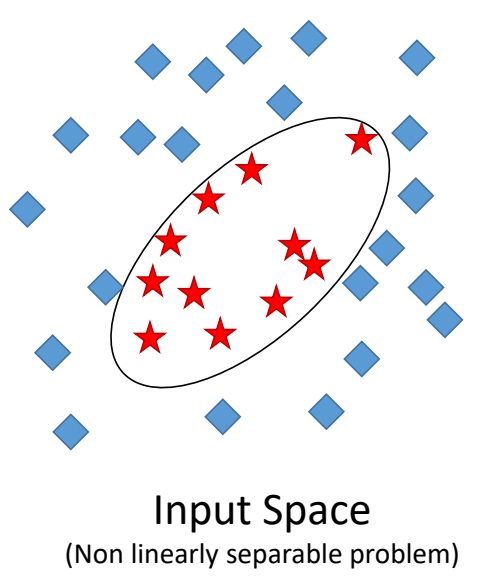

Higher dimensional feature space (Linear separating hyperplane)
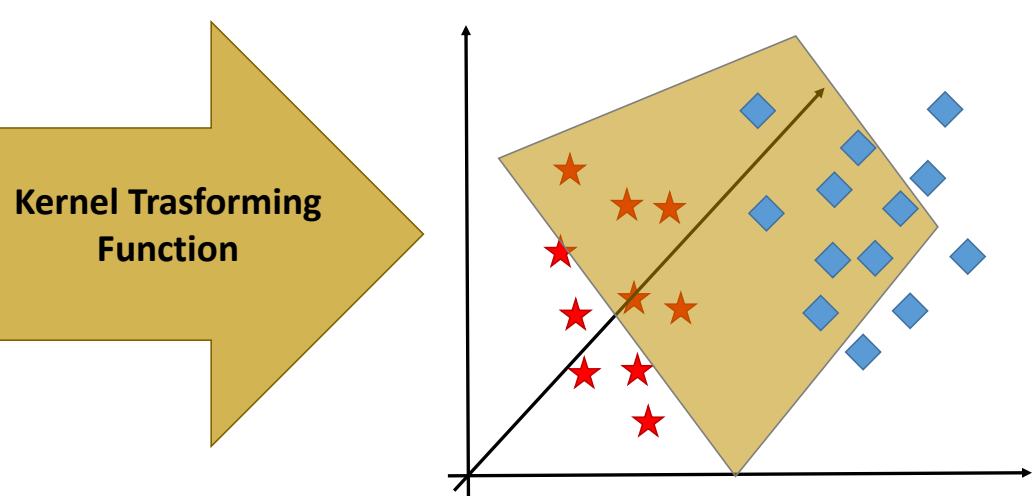

Figure 2. The basic principle of the SVM approach to classification: the projection to a higher dimensional space with a kernel and the identification of the best separating hyperplane.

dimensional feature space, an optimal separating hyperplane is constructed in order to minimize the risk of misclassification. The minimization of the error risk is obtained by maximizing the margins between the hyperplane and the closets points, the support vectors, 
of each class, as shown in Figure 1. This is achieved by a careful selection of the constraints of a suitable functional to maximize. The hyperplane is determined by a subset of points of the two classes, named Support Vectors (SV). SVMs therefore basically consist of suitable kernels, which project the inputs into higher dimensional spaces, where the classification becomes a linearly separable problem and can be solved with traditional quadratic programming methods with linear constraints based on the Lagrange multipliers. The main ideas behind the SVM approach are illustrated in Figure 2.

In mathematical terms, given a training set of $\ell$ samples $\left(\mathbf{x}_{1}, y_{1}\right), \ldots,\left(\mathbf{x}_{\ell}, y_{\ell}\right), x_{i} \in \mathfrak{R}^{n}$, for a binary classification problem such as disruptive or non disruptive discharges (i.e. $\left.y_{i} \in\{+1,-1\}\right)$, SVM estimates the following decision function:

$$
d(\boldsymbol{x})=\sum_{i=1}^{\ell} \alpha_{i} y_{i} H\left(\boldsymbol{x}_{i}, \boldsymbol{x}\right)
$$

where $H\left(\mathbf{x}_{i}, \mathbf{x}\right)$ is a kernel function and the parameters $\alpha_{i}, i=1, \ldots, \ell$ are the solutions of the following quadratic optimization with linear constraints:

maximization of the functional

$$
Q(\alpha)=\sum_{i=1}^{\ell} \alpha_{i} \alpha_{j} y_{i} y_{j} H\left(\boldsymbol{x}_{i}, \boldsymbol{x}_{j}\right)
$$

subject to the constraints

$$
\sum_{i=1}^{\ell} y_{i} \alpha_{i}=0, \quad 0 \leq \alpha_{i} \leq \frac{c}{\ell}, i=1, \ldots, \ell
$$

where $C$ is a regularization parameter [15].

The data points $\mathbf{x}_{i}$ associated with nonzero values of the coefficients $\alpha_{i}$ are called support vectors, which give the name to the technique. Once the support vectors have been determined, the SVM boundary between the two classes can be expressed in the form

$$
d(\boldsymbol{x})=\sum_{\text {vectors }}^{\text {support }} \alpha_{i} y_{i} H\left(\boldsymbol{x}_{i}, \boldsymbol{x}\right)
$$

$d(\mathbf{x})$ is the distance from the input $\mathbf{X}$ to the hyper-plane that separates the two classes and, hence, the hyper-plane points satisfy $d(\mathbf{x})=0$.

The rule to classify a feature vector $\mathbf{u}$ as non-disruptive (class $d_{\text {Safe }}$ ) or disruptive (class $d_{D i s}$ ) is given by: 


$$
\begin{aligned}
& \text { if } \operatorname{sgn}(D(\boldsymbol{u})) \geq 0 \\
& \boldsymbol{u} \in C_{D i s r} \\
& \text { otherwise } \\
& \boldsymbol{u} \in C_{\text {Safe }}
\end{aligned}
$$

where $\operatorname{sgn}(t)$ is the sign function.

Given the structural stability that they achieve by implementing the margins, SVMs are very powerful tools and have performed extremely well in the case of disruption prediction, as demonstrated in real time by APODIS $[9,10]$. Their hyperplane can therefore be considered a very good approximation of the boundary between the disruptive and not disruptive regions of the operational space.

\subsection{Probabilistic SVM}

Developing classifiers, which can provide a calibrated posterior probability, is extremely useful for subsequent post processing. For example, associating a probability to the classification estimate is fundamental for deploying utility models. Posterior probabilities are also indispensable when, as in our case, it can be important to combine the classifier output with other tools in an integrated decision support system (DSS). Unfortunately, traditional SVM do not provide a probabilistic output. Their basic version has therefore to be extended to associate a probability to their classification. Various methods have been proposed to modify the SVM so that they yield a probability [16]. The approach, adopted in this paper, is based on the classification performed by the SVM, to which a probability is associated by making recourse to the Bayes rule according to the formula:

$$
P(y=1 \mid f)=\frac{p(D \mid y=1) P(y=1)}{\sum_{i=-1,1} p(D \mid y=i) P(y=i)}
$$

In equation (5) $\mathrm{P}(\mathrm{y}=1)$ is the prior probability of class 1 and $\mathrm{p}(D \mid \mathrm{y}=1)$ indicates its likelihood where $\mathrm{D}$ is as usual the distance to the hyperplane. Therefore two quantities have to be determined to apply equation (5): the prior probability and the likelihood. In our application, the natural choice of the prior probability is the percentage of time slices seen so far in the campaign for the class to which the SVM classifies the new example. The main 
issue in relation (5) resides therefore in the determination of the likelihood. To this end, a class of methods comprises the techniques, which define a neighbourhood of each new point to classify and determine the percentage of the examples, belonging to the class selected by the SVM, in that neighbourhood [16]. Theoretical and numerical considerations have shown that for our application one alternative advantageous solution consists of remapping the distance to the hyperplane to a probability by using a sigmoid function, which has the right mathematical properties to be a probability:

$$
P(y=1 \mid D)=\frac{1}{1+\exp (A d+B)}
$$

In equation (6), $\mathrm{A}$ and $\mathrm{B}$ are two fitting parameters, and again $d$ indicates the distance of the examples to the SVM hyperplane. The determination of the likelihood function therefore takes place after the training. The examples in the training set, or in a hold out set, are classified and their distances to the separating hyperplane are used to fit the parameters of the sigmoid (6). The sigmoid is typically centred on the hyperplane, in the sense that points at distance zero from it have $50 \%$ probability of being in any of the two classes (see Figure 3). To summarise, for each new case, the class is determined by the SVM and the prior by the percentage of cases belonging to that class detected up to the current example.

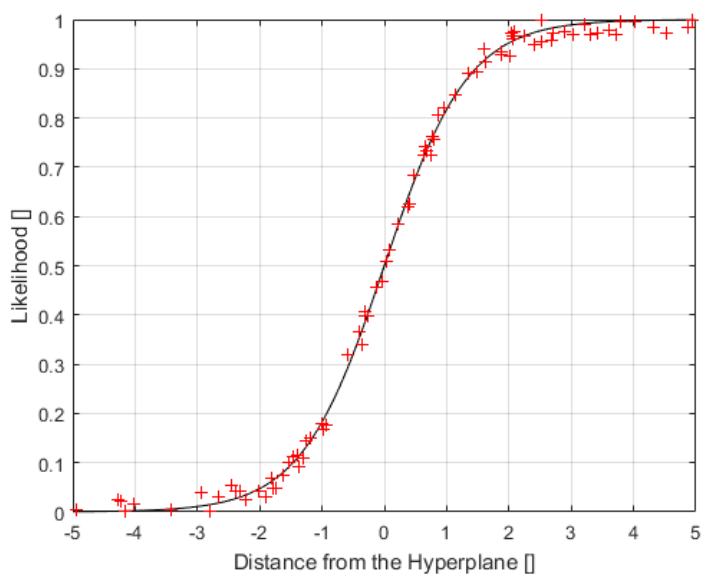

Figure 3. Sigmoid fit of the distance $d$ to the hyperplane to determine the likelihood.

The likelihood is computed using the sigmoid, which, together with the prior, allows calculating the probabilistic output according to the Bayesian equation (5). The resulting posterior probability is strictly speaking the level of confidence in the prediction that the discharge is going to disrupt. 


\section{Training of the Adaptive Probabilistic Predictor and JET Databases}

This section provides the details about the method used to train the probabilistic predictors (Subsection 3.1) and provides a general overview of JET databases to which they have been applied (Subsection 3.2). Information about the computational requirements is provided in Subsection 3.3.

\subsection{Training of the adaptive predictor from scratch}

As already mentioned, large devices might not have the luxury of collecting many disruptive examples to train machine learning predictors with the traditional data mining algorithms. It is therefore important to devise training approaches from scratch, which can start predicting with a limited amount of examples; they have also to be sufficiently adaptive to preserve good performance as the campaigns progress.

Various approaches can be adopted to train an adaptive predictor. In harmony with previous instances [9-12], a quite simple strategy has been implemented for the training. The predictors are designed with a "from scratch" approach and therefore need only one disruptive and one non disruptive case to build the first model. In the campaigns analysed, the first disruption occurred after a while and therefore the first model was obtained after the first disruption. For the disruptive discharge, $15 \mathrm{~ms}$ before the beginning of the current quench have been divided in 5 intervals of $3 \mathrm{~ms}$ each and the averages of the selected signal features over these three intervals have been used as input to the training. The 5 discharges prior to the first disruptive one have been used as examples for the safe case. For each of these discharges, two random periods of $20 \mathrm{~ms}$, with plasma current above $750 \mathrm{kA}$, have been averaged and the averages over these intervals have been used as inputs for the training. The number of examples can be increased to improve performance but at the expenses of computational resources for the SVM training (which is a quite heavy process). For the campaigns whose results are presented in the paper the choice of two training points is more than adequate as will become apparent in the following.

The model derived as previously described needs to adapt in two different directions by learning how a) disruptions vary and b) the safe space of operation changes. In other words, the model has to be automatically updated to follow the evolution of the boundary between the disruptive and non disruptive regions of the operational space as the experimental campaigns evolve. To this end a model, starting with the one obtained after the first training described above, is used for the following discharges until the first missed alarm. When the previous model misses a disruption, the shot not properly classified is 
included in the training set. In this way a new model is determined, which is deployed to analyse the following discharges until the next error, which provides an example for a new retraining.

If the error is a false alarm, it is not appropriate to insert that example in the training set and retrain the predictor. In closed loop real time applications, indeed, it is not necessarily the case that false alarms can be always recognised, after the discharge has been shutdown following the received alarm. Of course post pulse investigations by the experts and analysis of possible technical faults can provide indications that the discharge was stopped prematurely, but this cannot be assumed to happen systematically. On the other hand, retraining only on the basis of disruptive examples can cause the number of false alarms to increase unnecessarily, particularly during the course of long campaigns and/or when the scenarios evolve and new regions of the operational space are explored. As a compromise solution, adopted to obtain the results described in the following, the retraining has been performed with a new safe example every time the model launches an alarm. After each alarm the previous discharge, if safe as it is normally the case, is used as an example of a non disruptive discharge to retrain. Now for the training two $10 \mathrm{~ms}$ intervals, around the maximum value of the locked mode, have been averaged and these averages are the features for the new training.

Of course, in the case of closed loop applications of the predictors, more sophisticated strategies could be implemented, such as retraining when new scenarios are developed and run or by identifying some false alarms. Therefore the results reported in the following, even if quite good, have to be considered an underestimate of the possible performance in terms of false alarms. Indeed the proposed approach can be run automatically but if man power is available to optimise the training, the percentage of false alarms can be easily reduced by a factor. This can be easily proven by correcting the first false alarms in the adaptive training, which reduces the total false alarms to about $2 \%$.

With regard to the implementation strategy, another aspect must be explicitly considered. Given the fluctuations of the various plasma parameters, triggering an alarm after the first time slice, when the models predict a disruptive behaviour, is not necessarily the best strategy. To increase reliability, it would be better to wait for the model to predict a disruption for two or three subsequent time intervals. This is the traditional choice implemented when using a traditional SVM, which classifies giving only a binary output, the class the example belong to. Now the probabilistic output provides an additional knob to work with, the probability threshold; therefore the question can be asked whether an optimal 
trade-off exists between using a different threshold and waiting some time before triggering an alarm. This optimization problem has been investigated in detail as reported in the sections 4 and 5 .

\subsection{JET Databases: ITER Like Wall and Carbon wall}

In building both databases, the intentional disruptions have been eliminated from the training. Indeed they do not need to be predicted and, being typically different from naturally occurring disruptions, can affect the quality of the adaptive training. Of course, they have also been excluded from the computation of the final statistics (see next sections). Only discharges whose plasma current exceeds $750 \mathrm{kA}$ have been considered but no other general selection has been implemented. All the signals have been resampled at $1 \mathrm{kH}$ frequency. Alarms, which are launched $10 \mathrm{~ms}$ or less from the beginning of the current quench, are considered tardy, since $10 \mathrm{~ms}$ is the minimum time required on JET to undertake mitigation action. Alarms triggered more than $2.5 \mathrm{~s}$ before the beginning of the current quench are considered early, even if this choice is a bit penalising because in various instances, indeed, the predictors have detected an almost disruptive situation but the plasma just managed to survive longer than $2.5 \mathrm{~s}$. Therefore if an alarm had been launched in these cases, since the quality of the plasmas had already been compromised, in general no useful experimental time would have been lost and time for a soft landing would have been available. Therefore keeping these cases in the list of the not properly classified discharges is a conservative choice.

Coming to the database with the ILW wall, the campaigns C29 to C31 have been
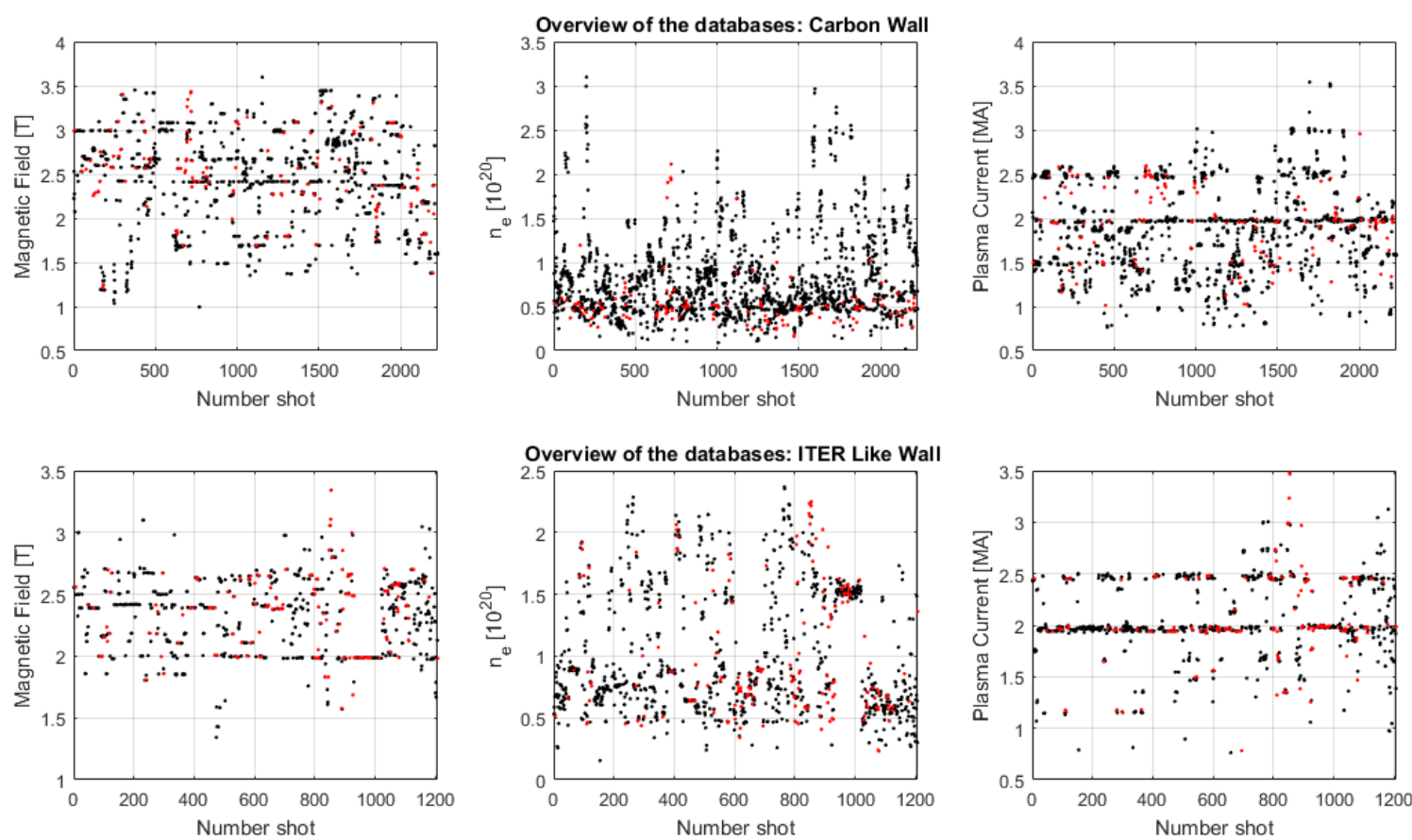

Figure 4 .Overview of the databases for the Carbon wall and ILW- A characteristic point for each shot in the database has been reported. The red points belong to disruptive shots. 
considered. After proper cleaning and validation of the database, overall 187 disruptive and 1020 non disruptive shots are included. A plot showing the operational space covered by the database is shown in Figure 4 (bottom row).

The database of the $\mathrm{C}$ wall comprises the campaigns C15a, C15b, C16, C1617, C18, C19 (from shot 65988 to 70749). Overall, 143 disruptive and 2083 non-disruptive shots are included. A plot showing the operational space covered by the database is shown in Figure 4 (top row).

\section{$\underline{3.3 \text { Computational aspects }}$}

With the adaptive approach implemented, and described in detail in subsection 3.1, the computational requirements for the deployment of the proposed tools are very limited. The training, which is the computationally most demanding phase of a predictor development, typically involves a quite limited number of discharges and therefore can easily be performed between shots. Once the model is available, as already demonstrated for the case of APODIS, the classification of a new time slice can be easily performed in a fraction of JET system cycle time of $2 \mathrm{~ms}$ [19]. The proposed tools are therefore fully compatible with real time applications. The retraining can easily be performed between shots and their deployment in real time would provide an instantaneous response on the time scale of typical feedback controls systems in Tokamaks.

\section{Results for disruption mitigation: JET with an ITER Like Wall}

To show the potential of the approach compared to previous tools, it has been decided to select the amplitude of the locked mode and the internal inductance signals as inputs. These are indeed quantities routinely available in real time in JET and have been used since a

Table I. Main indicators of MAPPsvm quality using only the SVM output to decide whether to trigger an alarm. The three rows show the results for the cases when one single disruptive time slice is considered sufficient to trigger an alarm and when, on the contrary, two or three consecutive time slices are required.

\begin{tabular}{|l|c|c|c|c|c|c|c|}
\hline SVM & $\begin{array}{c}\text { Success } \\
\text { Rate \% }\end{array}$ & Missed \% & Early \% & Tardy \% & False \% & $\begin{array}{l}\text { Mean } \\
{[\mathrm{ms}]}\end{array}$ & $\begin{array}{l}\text { Std } \\
{[\mathrm{ms}]}\end{array}$ \\
\hline $\begin{array}{l}\text { 1 Disurptive } \\
\text { time slice }\end{array}$ & 94.62 & 0.54 & 2.69 & 2.15 & 5.58 & 321 & 332 \\
\hline $\begin{array}{l}\text { 2 Disurptive } \\
\text { time slices }\end{array}$ & 95.16 & 0.54 & 2.15 & 2.15 & 5.79 & 320 & 344 \\
\hline $\begin{array}{l}\text { 3 Disurptive } \\
\text { time slices }\end{array}$ & 94.62 & 0.54 & 2.15 & 2.69 & 5.79 & 325 & 337 \\
\hline
\end{tabular}


long time to develop machine learning predictors. Moreover, recent theoretical considerations seem to indicate that these to quantities are the main ingredients for multimachine modelling of the onset of disruptions [20]. To start, the adaptive "from scratch" training has been performed using the simple output of the SVM, not the posterior probability, to decide about

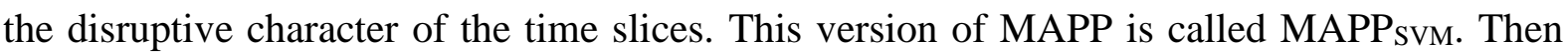
the possible improvements in performance, which can be achieved by selecting an optimal threshold in the posterior probability, have been investigated. For the time to trigger an alarm, three different choices have been tested: immediately after the first time slice the predictor detects a disruptive behaviour, immediately after two consecutive time slices and immediately after three consecutive time slices.

The overall quality of the predictor, using only the output of MAPP $\mathrm{SVM}_{\mathrm{S}}$ to take the decision whether or not to trigger an alarm, can be appreciated by inspecting Table I, which reports the main figures of merit used to assess disruption predictors. The success rate of the predictor is quite good and the false alarm rate more than satisfactory. This is particularly remarkable since it is to be remembered that MAPP is an adaptive predictor and therefore it is trained during the same campaigns in which its performance are evaluated.

Table II. Main indicators of MAPP quality using the posterior probability to decide whether to trigger an alarm. The threshold of $80 \%$ provides a good trade-off between success rate and false alarms and therefore it is the reference case discussed in the rest of the paper.

\begin{tabular}{|c|c|c|c|c|c|c|c|}
\hline $\begin{array}{c}\text { Threshold } \\
\text { post prob } \\
\text { DISR }\end{array}$ & $\begin{array}{c}\text { Succes } \\
\text { Rate \% }\end{array}$ & Missed \% & Early \% & Tardy \% & False \% & $\begin{array}{c}\text { Mean } \\
{[\mathrm{ms}]}\end{array}$ & $\begin{array}{c}\text { Std } \\
{[\mathrm{ms}]}\end{array}$ \\
\hline $30 \%$ & 87.63 & 0.54 & 10.21 & 1.61 & 12.67 & 336 & 338 \\
\hline $40 \%$ & 93.55 & 0.54 & 4.30 & 1.61 & 6.45 & 338 & 339 \\
\hline $50 \%$ & 95.70 & 0.54 & 2.15 & 1.61 & 5.69 & 329 & 339 \\
\hline $60 \%$ & 96.23 & 0.54 & 1.61 & 1.61 & 5.40 & 330 & 338 \\
\hline $70 \%$ & 97.31 & 0.54 & 0.54 & 1.61 & 5.12 & 331 & 338 \\
\hline $80 \%$ & 97.31 & 0.54 & 0.54 & 1.61 & 4.62 & 316 & 333 \\
\hline $90 \%$ & 96.23 & 0.54 & 0.54 & 2.69 & 4.23 & 317 & 330 \\
\hline
\end{tabular}

Notwithstanding the good performance of MAPPSvM, it is essential to see whether the performance can be improved by selecting a proper threshold in the posterior probability. A systematic investigation of this aspect has been performed. MAPPSvm has been rerun in an adaptive way for different levels of posterior probabilities and the different choices for the 
number of consecutive time slices for which the threshold is exceeded. The results are reported in Tables II for the case of the alarm triggered immediately after the first time slice considered disruptive by this version of MAPP. In appendix A, Tables A1 and A2 report the

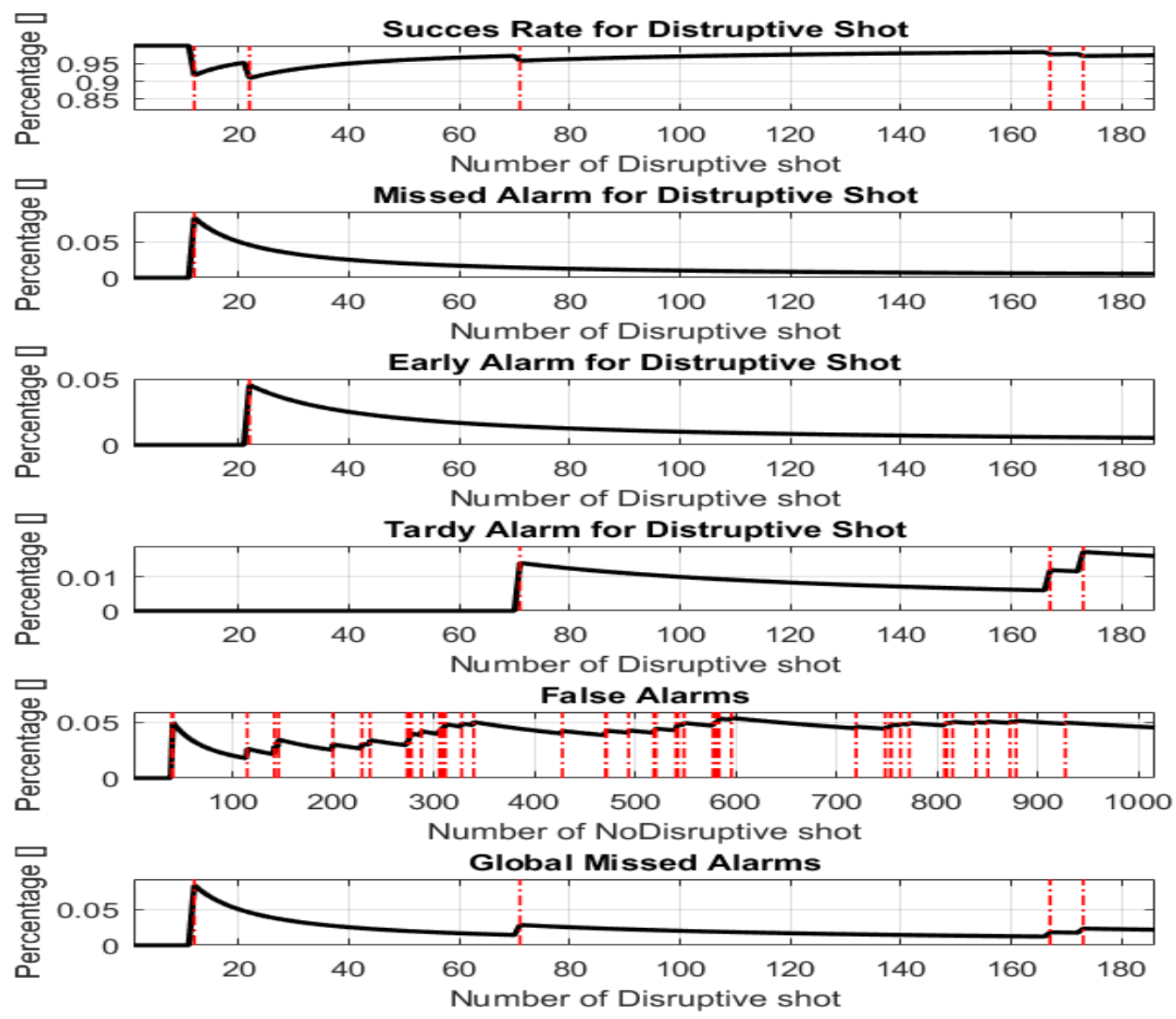

Figure 5. MAPP $1,80 \%$ : evolution of the figures of merit in the analysed campaigns. The vertical red lines indicate the shots where an error has occurred.

cases for which two and three consecutive disruptive time slices are required to trigger an alarm. From the tables it is evident that, even if the results obtained by MAPPsvm are already quite good, an optimum of the posterior probability to maximise performance exists. If the threshold is chosen as $80 \%$ and an alarm is triggered after the first time slice crosses this value of the probability, a quite good compromise if found between missed alarms and false alarms is minimised. This version of MAPP is referred to as $\mathrm{MAPP}_{1,80 \%}$ in the following. The evolution of $\mathrm{MAPP}_{1,80 \%}$ performance of is shown graphically in Figure 5. The performance of the predictor is quite positive and would satisfy the needs of the next generation of devices such as ITER. The quality of $\mathrm{MAPP}_{1,80 \%}$ are even more remarkable if one considers the actual 
behaviour of the so called tardy alarms. It has been checked that they all occur because, in these cases, the first symptoms of disruptions appear later than $10 \mathrm{~ms}$ before the beginning of the current quench. An example of these cases is reported in Figure 6, from which it can be seen that the inputs to the predictor do not give any hint about an incoming disruption before
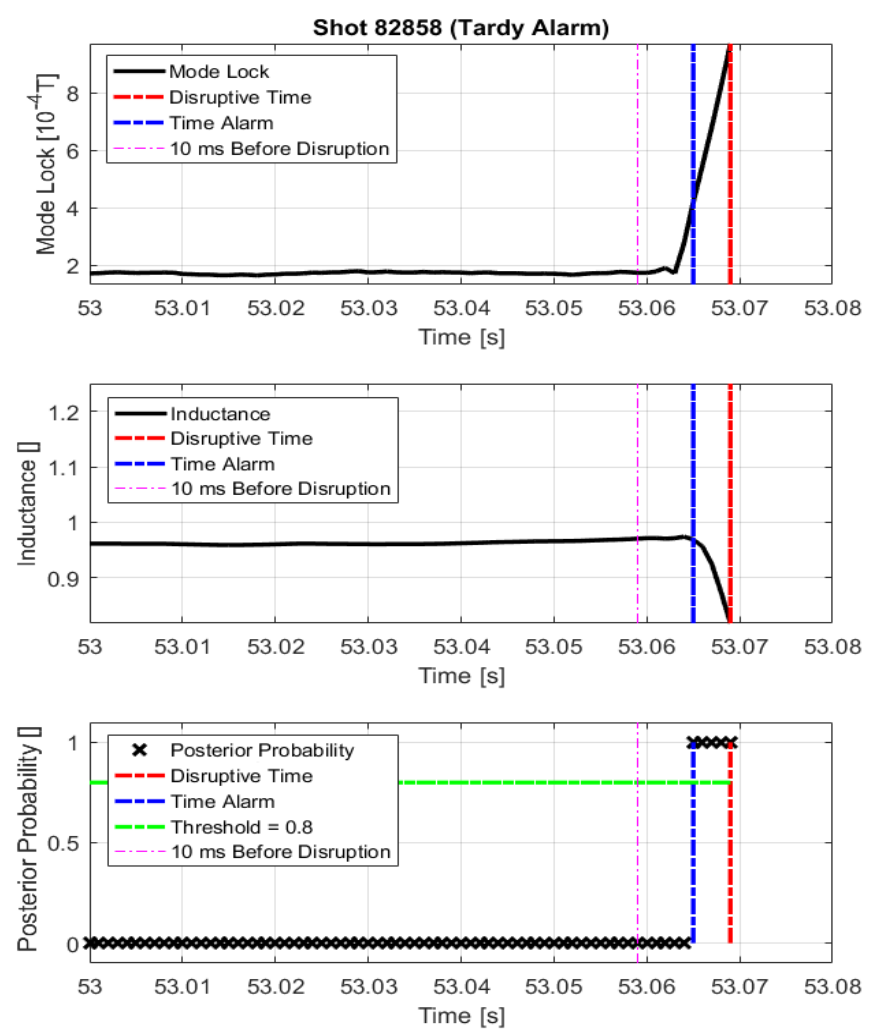

Figure 6. Evolution of the signals input to MAPP1,80\% for the case of a tardy alarm. From top to bottom: evolution of the locked mode, evolution of the internal inductance and of the disruption probability.
$10 \mathrm{~ms}$ from the beginning of the current quench. As soon as the signals indicate an anomalous behaviour, the probability of disruption immediately jumps from zero to $100 \%$. Of course, if the signals do not show any sign of disruption, in a certain sense the predictor is justified in not triggering an alarm. If the definition of tardy detection is moved later, progressively all the tardy disruptions are detected. If this aspect is taken into account, it can be seen that the number of missed alarms after the first training is always of the order of one or two shots. Therefore it is possible to argue that developed predictor

exploits all the information in the signals [21].

Since the number of false alarms is about $2.5 \%$, the predictor performs acceptably for ITER and it is difficult to expect a major improvement in performance using the locked mode and inductance as inputs. It is also worth noting that the predictor has mainly to learn the safe behaviour; much more retraining is indeed required due to false alarms whereas only a handful of cases are enough for MAPP to identify disruptions. This corresponds to the experimental practice, since it is normal that, during campaigns, the configurations are changed and progressively pushed to explore new regions of the operational space. The evolution of the operational space requires an adaptive predictor to adjust and take into account the new experimental situations. 

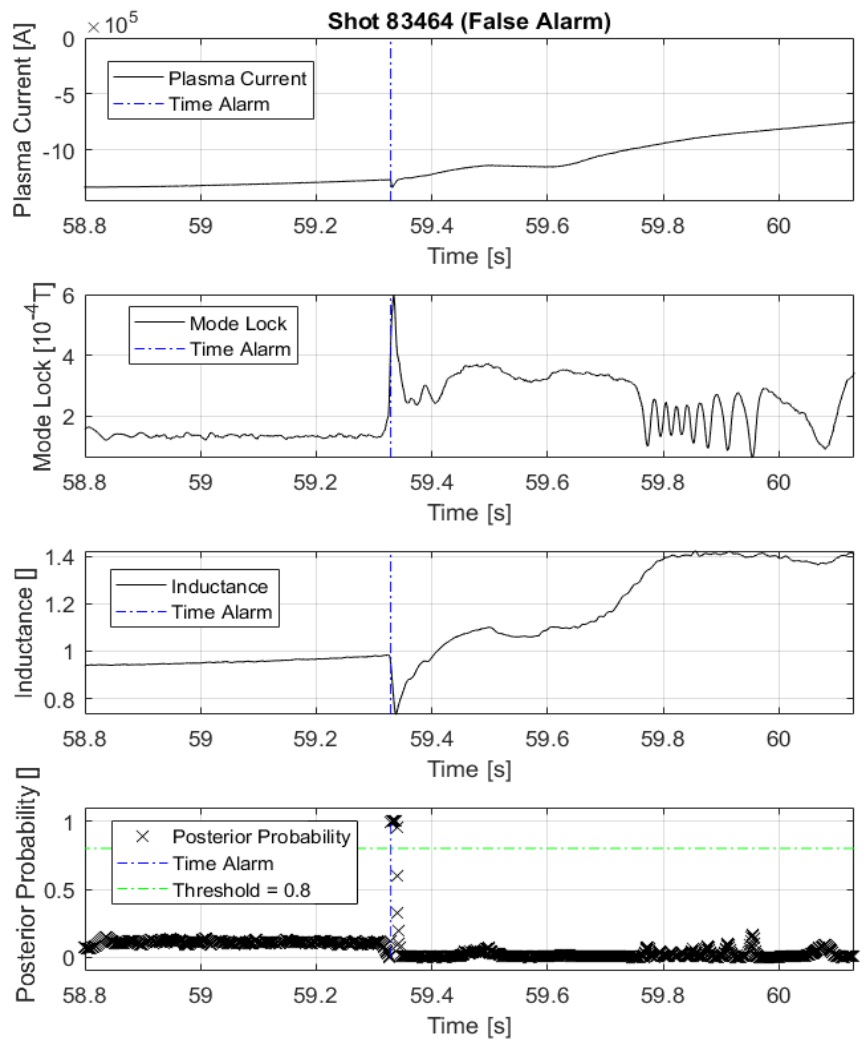
Figure 7. Evolution of the signals input to current ramp down a current MAPP $1,80 \%$ for the case of a dubious false alarm.
With reference to the false alarms, it is worth mentioning that the statistics shown in table II are also quite pessimistic. In addition to the fact, already mentioned, that the adaptive training from scratch has been performed blind, without any a posteriori information, it is to be noticed that some false alarms included in the statistics are really at the limits. Many of them are considered false alarms only because the current does not reach the decay rate of $5 \mathrm{MA} / \mathrm{s}$, considered in JET the threshold to declare a quench. The reported numbers in Table II respect this convention even when it is very questionable. A typical example is reported in Figure 7 , in which a jump in the locked mode appears exactly at the beginning of the current ramp down. The control system manages to keep the current decay below the threshold of $5 \mathrm{MA} / \mathrm{s}$ but it is very dubious that, by detecting an anomaly, the predictor really made a mistake in this case.
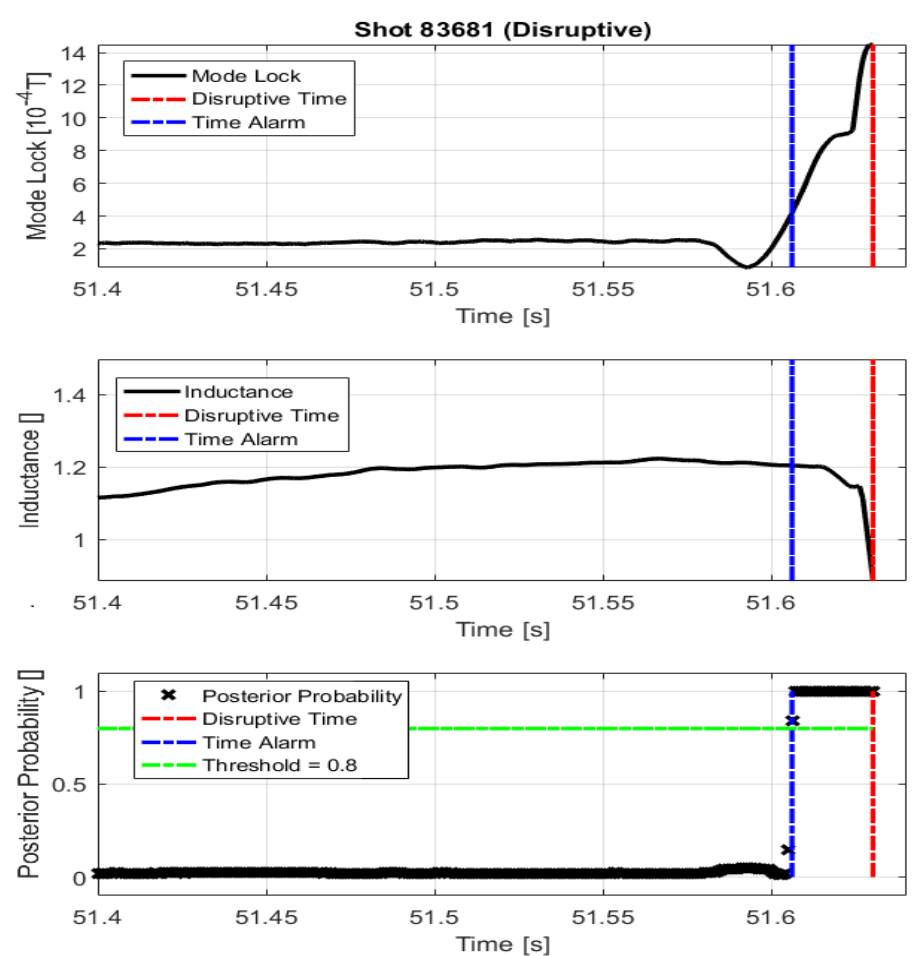

Figure 8. Evolution of the signals input to $\mathrm{MAPP}_{1,80 \%}$ for the case of a typical disruption. 
A part form the excellent $\mathrm{MAPP}_{1,80 \%}$ performance, the results of the scan in probability deserve some comments. The fact that performance does not vary proportionally to the variations in the probability threshold is due to the behaviour of the input signals, in
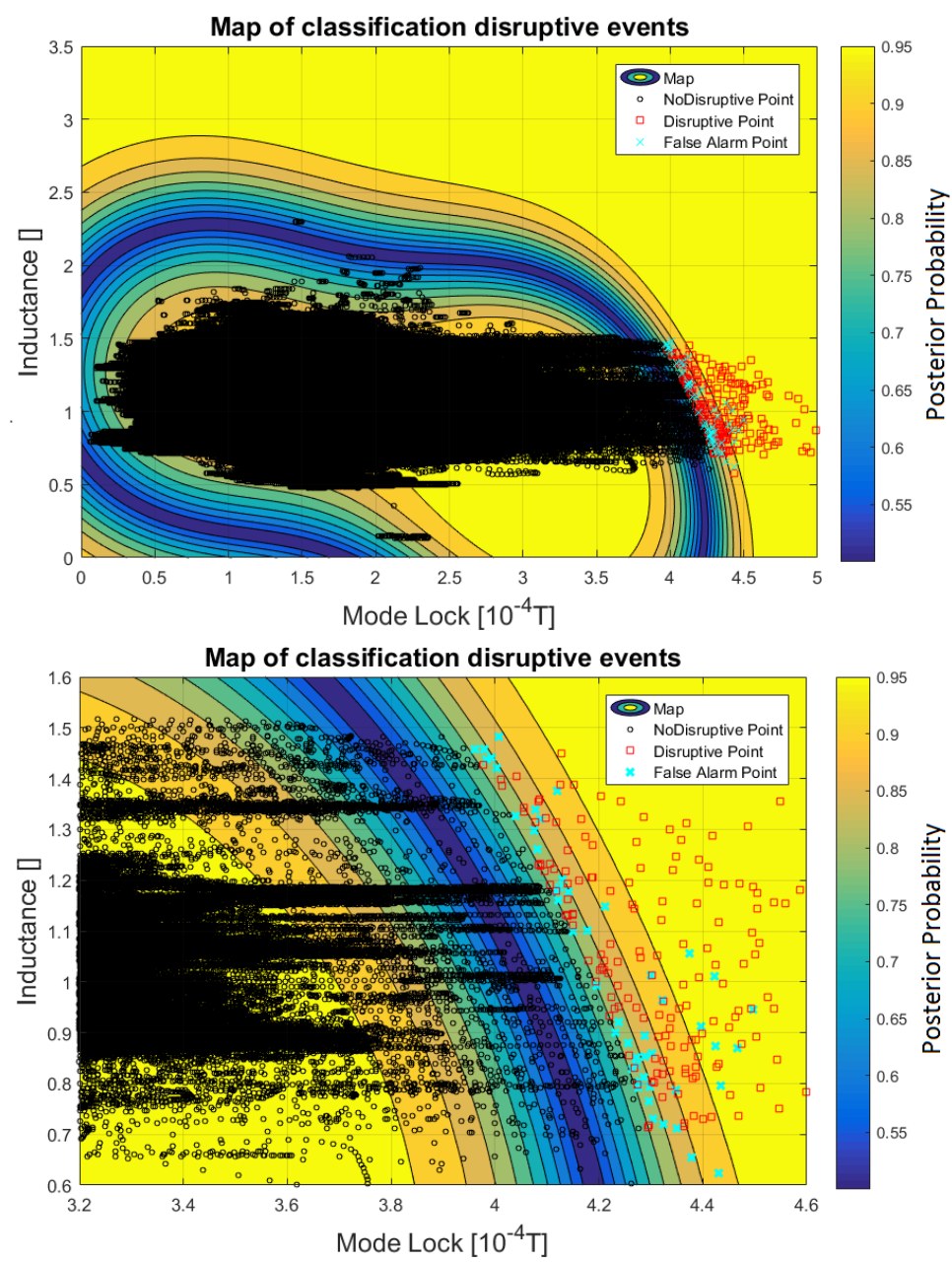

Figure 9. Top: plot of the safe and disruptive regions of the operational space in JET with the ILW as obtained with MAPP $1,80 \%$. The colour codes indicates the value of the posterior probability. The black crosses are all the nondisruptive shots (all time slices for each shot at $1 \mathrm{~ms}$ time resolution). The red circles are the data of the disruptive shots at the time slice of the alarm. Bottom: zoom of the most relevant boundary region. particular the locked mode. Indeed, as shown in the first plot of Figure 8, for a typical disruptive discharge the locked mode signal changes so abruptly that the probability of disruption increases from almost $0 \%$ to almost $100 \%$ in a matter of a single time slice (time scales of about $1 \mathrm{~ms}$ ). This fact limits the impact of selecting a different probability threshold for triggering an alarm. Moreover, since the aforementioned behaviour 
is quite general, the choice of the locked mode signal seems to be questionable for the purpose of devising a proportional reaction to alarms of various intensities for avoidance. The
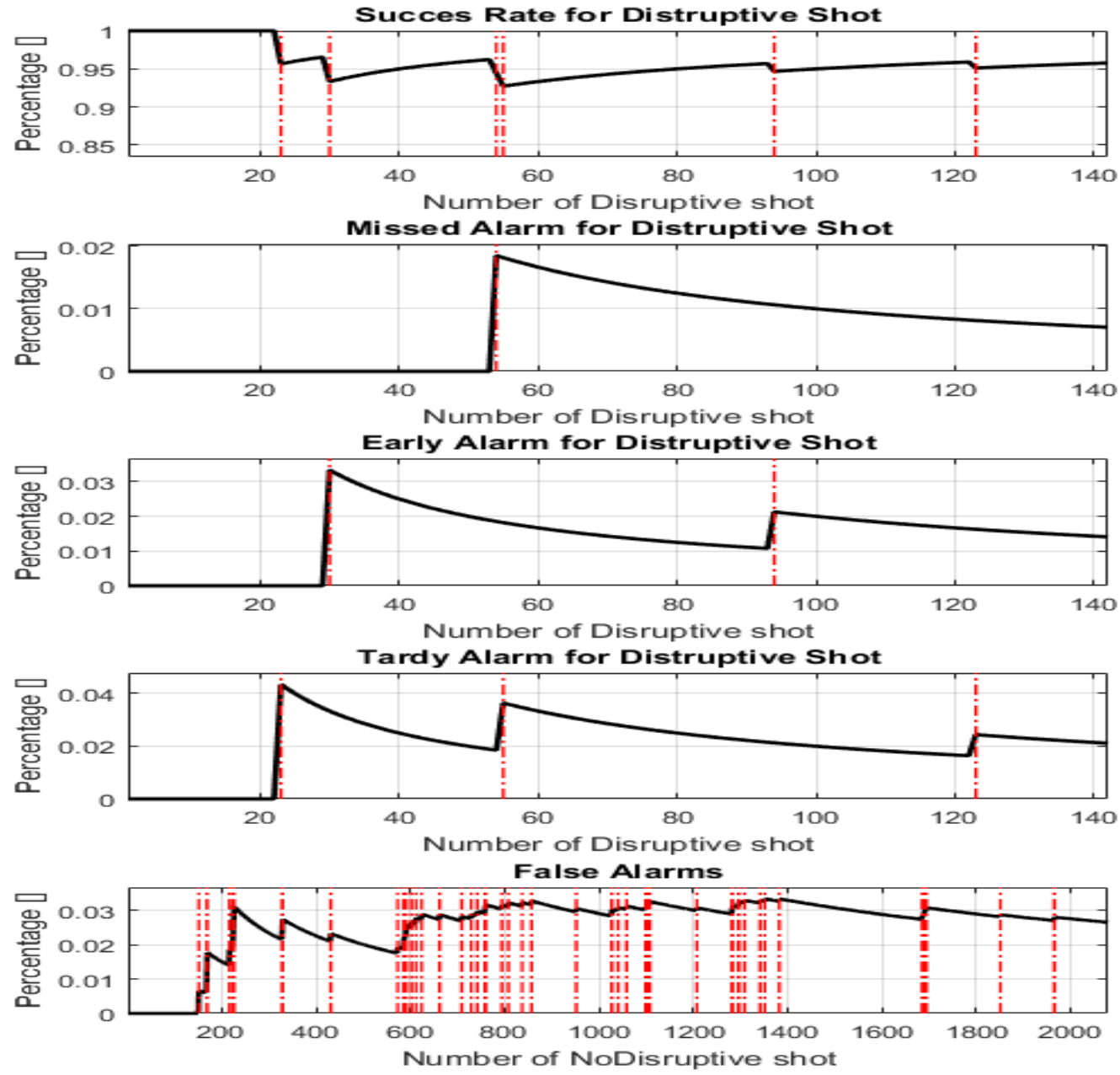

Figure10. MAPPCarbon: evolution of the figures of merit in the analysed campaigns. The vertical red lines indicate the shots where an error has occurred.

problem is further complicated by the fact that the locked mode precursor can manifest itself in a wide interval of times before the beginning of the current quench, from the order of a few seconds to a few ms. This is the main reason why predictors based on the locked mode signals have a dispersion in the warning times, which is typically very large, as confirmed from the previous tables. Therefore the locked mode signal seems to be appropriate as an input for last resort predictors aimed at triggering mitigation but not for avoidance.

A second important observation is based on the evolution of the boundary between disruptive and non-disruptive parts of the operational space, as plotted on the feature space of locked mode and internal inductance (see Figure 9). The transition between the safe and disruptive regions is a curve and a good separation cannot be achieved by selecting a simple threshold on the locked mode, as done in predictors such as LMPT. A threshold in the locked 
mode signal would correspond to a vertical line in the plot of Figure 9. A good separation between the safe and disruptive regions of the operational space cannot be achieved with such a threshold. Indeed from the plot of Figure 9 it is evident that the threshold in the locked mode signal depends on the value of the plasma inductance. This observation contributes to explaining why on JET predictors based on a threshold in the locked mode have traditionally performed worse than multisignal classifiers such as APODIS [21].

\section{Results for disruption mitigation: JET Carbon Wall}

To confirm the general applicability of the method described in the previous sections, the same approach has been applied also to a database of disruptions of JET with the carbon wall. Again, the amplitudes of locked mode and the internal inductance signals have been selected as inputs. The same adaptive training procedure, described in detail in Section 3.1 has been implemented. This version of the predictor is called MAPP ${ }_{\text {Carbon. The same criteria }}$ to evaluate the performance as used for the ILW case have been adopted. The results, in

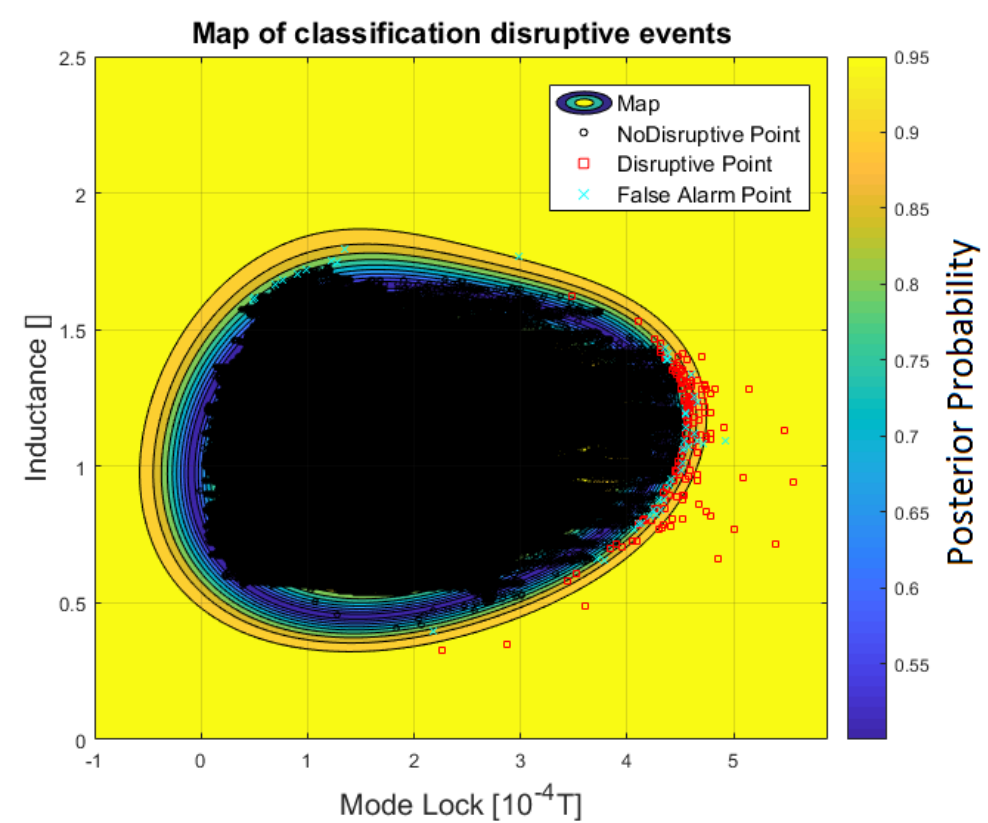

Figure 11. Plot of the safe and disruptive regions of the operational space in JET with the Carbon wall as obtained with MAPPCarbon (threshold at $\mathbf{8 0 \%}$ ). The colour codes indicates the value of the posterior probability. The black crosses are all the non-disruptive shots. The red circles are the data of the disruptive shots at the time of the alarm. terms of the traditional basic indicators used to qualify predictors, are reported in Appendix B. Also in this case, a good equilibrium between success rate of disruption detection and false alarms is achieved for a probability threshold of $80 \%$ and triggering after the detection of the first disruptive time slice. For this level of threshold the success rate is almost $96 \%$ and the false alarm are $2.6 \%$. With regard to these very competitive results, the

same considerations, mentioned for the case of the ILW, also apply. The statistics are 
conservative both in terms of success rate and false alarms and for the same reasons. A graphic summary of $\mathrm{MAPP}_{\mathrm{Carbon}}$ performance, emphasizing the temporal evolution of the results is provided in Figure 10.

From the tables in Appendix B and the plots of Figure 10 it is clear that the proposed methodology provides very good results also for JET with the carbon wall. This analysis therefore confirms the general character of the proposed technique, which can be applied to completely different experimental situations. The shape of the boundary in the plane of the Locked Mode and the Internal Inductance is reported in Figure 11. From the plot of the posterior probability, it is easy to see how the boundary between the safe and disruptive regions of the operational space is more involved in the case of JET with the carbon wall. This reflects the more forgiving nature of the wall, which allowed more freedom in experimenting than with the more delicate ILW.

\section{Application to disruption avoidance}

The results shown in this paper, and also those achieved by the most advanced general predictors deployed so far on JET, indicate that the performance obtained are not completely satisfactory. Tools such as the ones described in this paper can exploit practically the whole information contained in the input signals and they can be deployed for mitigation. Unfortunately, their performance are not good enough for avoidance. First, they cannot always provide sufficient warning times for undertaking successful avoidance actions, given the time scales of the current quench on JET and the response time of the available actuators. Second, the dispersion in the warning times remains too large, which constitutes a serious issue for the logic presiding on the decision about what actions to take. Indeed, at the moment

Table III. Comparison of the SVM using the locked mode and inductance and the SVM using the radiated fraction and the inductance as inputs

\begin{tabular}{|l|c|c|c|c|}
\hline \multicolumn{1}{|c|}{ Typology } & Percentage & $\begin{array}{c}\text { Discrete } \\
\text { Ratio }\end{array}$ & $\begin{array}{c}\text { Mean } \\
{[\mathbf{m s}]}\end{array}$ & $\begin{array}{c}\text { Std } \\
{[\mathbf{m s}]}\end{array}$ \\
\hline MAPP $_{\mathrm{PRAD}}$ False Alarms & 17.73 & $180 / 1015$ & --- & --- \\
\hline MAPP $_{1,80 \%}$ Success rate & 97.31 & $179 / 185$ & 316 & 333 \\
\hline Pre-Alarm MAPP $_{\mathrm{PRAD}}$ & 38.92 & $72 / 185$ & 860 & 528 \\
\hline
\end{tabular}

of the alarm, it is not possible to tell whether a disruption is imminent or whether there are 
hundreds of ms available to react. These of course are problems inherent in the characteristics of the signals used as input not of the predictor technology itself, as sown in Figure 8.

On the other hand, the classification tools described in the previous sections, being based on probabilistic SVM, can in principle be applied also to disruption avoidance and not only mitigation. Of course, such a strategy is predicated on the fact that signals can be found that give sufficient warning time and possibly vary more gently than the locked mode. If such signals are available, different actions can be undertaken depending on the value of the probability of disruption. In this section, an example is provided For JET with the ILW to illustrate the applicability and capability of the presented methodology. It must be emphasised that prediction for avoidance, and not for mitigation, is a new field of application for machine learning tools. Various aspects will have to be further investigated and more optimization remains to be implemented. Moreover, as described in detail in the following, new signals will have to be made reliably available in real time for the deployment of these new tools. In the rest of this section only an example to illustrate the potential of the

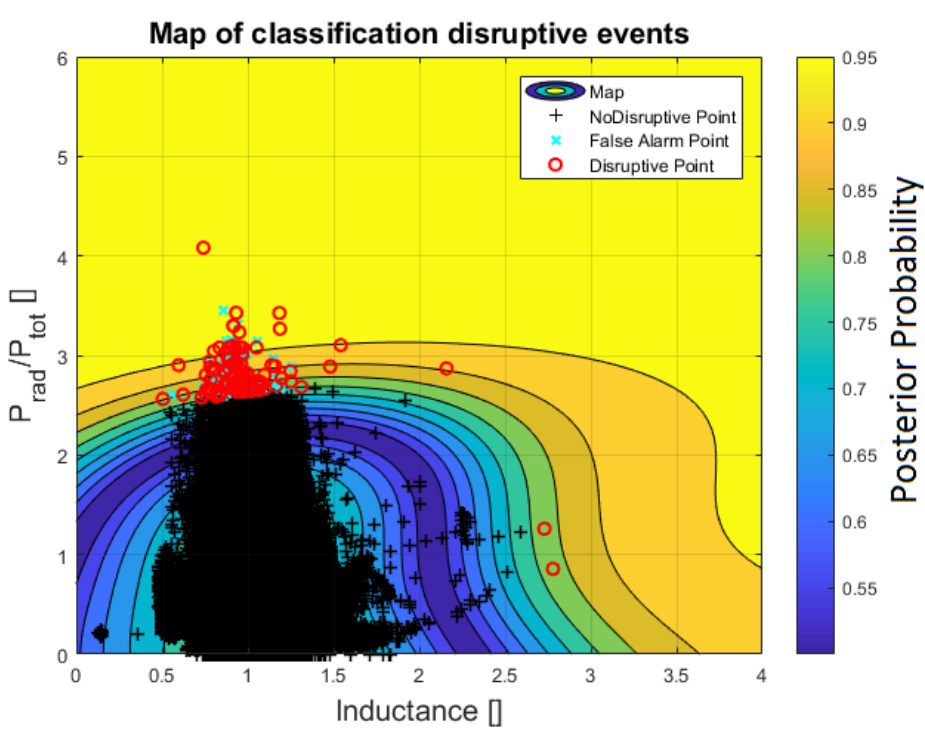

Figure 12. Plot of the safe and disruptive regions of the operational space in JET with the ILW as obtained with MAPPPRAD (threshold at $80 \%$ ). The colour codes indicates the value of the posterior probability. The black crosses are all the non-disruptive shots. The red circles are the data of the disruptive shots at the time of the alarm. developed tools is therefore provided.

In metallic machines, the control of heavy impurities is a major issue. Impurity accumulation can lead to disruptions. The radiation from the plasma can therefore be considered a potential indicator of the probability of disruptions. Moreover, it is expected that the dynamic of the radiated power is slower than the one of the locked mode. Therefore, it is worth

investigating whether the radiated fraction can help in prediction for avoidance. To this end, a probabilistic SVM has been trained (MAPP $\left.{ }_{P R A D}\right)$ using plasma internal inductance and the radiated fraction $\mathrm{P}_{\mathrm{RAD}} / \mathrm{P}_{\text {INPUT }}$ as inputs. The same adaptive procedure described in Section 3 has been followed again. 
The approach tested consists of comparing the SVM based on the locked mode and the internal inductance $\left(\mathrm{MAPP}_{1,80 \%}\right)$ as the basic predictor for mitigation and test to what extent the SVM using the radiated fraction can provide earlier alarms. Again, a threshold in probability of $80 \%$ provides the best compromise. The overall results are shown in Table III; as can be seen from this Table, there is an overall improvement in the warning time from about $360 \mathrm{~ms}$ to $860 \mathrm{~ms}$ over the whole set of disruptions. The standard deviation of the warning times is also more favourable if normalised to the mean. This comes at the price of an increase of false alarms to about $18 \%$, which can or cannot be acceptable depending on the campaign and the scientific objectives. The probability distribution of the disruptivity in the space of radiated fraction and
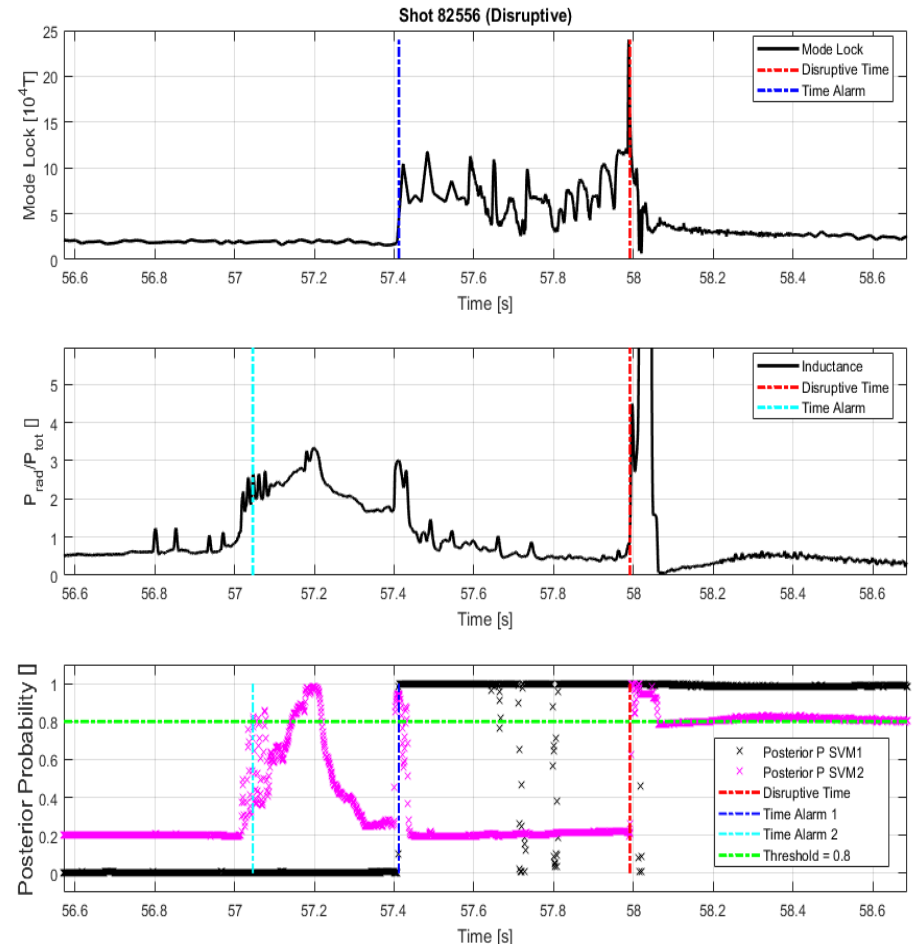

Figure 13. Evolution of the signals inputs to $\mathrm{MAPP}_{\mathbf{1 , 8 0} \%}$ and $M A P P_{R A D}$ and their output probability for a disruption due to a radiative collapse. The change in the disruption probability is much less abrupt for the predictor using the radiated fraction as input compared to the one using the locked mode.

internal inductance is shown in Figure 12.

Of course, since the predictors now give a probability as output, avoidance actions can be undertaken after an alarm from the SVM using the radiated fraction and then react according to the effects, always resorting to mitigation once the locked mode SVM launches an alarm. The radiated fraction, having slower dynamic evolution than the locked mode, allows some time for reaction, as can be seen from the evolution of the disruption probability for a radiative disruption shown in the plots of Figure 13. It is worth mentioning that he campaigns analysed do not include specific experiments aimed at testing the radiation limit; a specific investigation of the discharges devote to the operation at high radiated fraction is planned for the future using advanced tomographic inversion methods. 


\section{A probabilistic classifier for determination of disruption types}

As a consequence of the observations reported in the previous sections, probably one of the most promising strategies for the development of predictors for avoidance based on machine learning tools could consist of optimizing their performance for individual classes of disruptions. This consideration of course raises the question whether probabilistic SVM can also be deployed for the identification of disruption types. Indeed various approaches to the classification of disruptions have already been tested on JET data quite successfully. The most performing techniques are based on Self Organising Maps (SOM), Generative Topographic Mapping (GTM) and K-means with Geodesic Distance on Gaussian Manifolds. All these alternatives have provided very good results in terms of automatically determining the disruption type many tens of $\mathrm{ms}$ in advance of the beginning of the current quench $[22,23]$. On the other hand they do not provide naturally a calibrated probability as output. Therefore, it is natural to investigate the potential of the probabilistic SVM techniques to discriminate disruption types. This subsection is meant simply to provide an example of classification of the disruption types just to illustrate the potential of the developed technique. Classification is a completely new field for the SVM-based probabilistic predictors and therefore a more systematic treatment is required. In addition, it must be considered that the database available is not completely satisfactory; any different disruption types are seen on JET and their classification will require a much wider statistical basis. On the other hand, to prove the potential of MAPP type predictors to classify a simple example is sufficient.

As a feasibility tests, it has been decided to consider the cases when MAPPPRAD triggered an alarm. At the time of the alarm, a specific classifier has been developed to determine whether the disruption is due to excessive radiation. The objective of the classifier is therefore to determine whether, at the time of the alarm triggered by MAPPP ${ }_{\mathrm{RAD}}$, the disruption is caused by excessive radiation or other causes. This binary classifier is already a very ambitious task given the database. Indeed only a total of 72 shots are therefore available. The classification of the disruption type has been provided by the group of expert session leaders operating JET and it is considered completely correct. Of the 72 shots available, 10 (5 for each class) have been used for training and the remaining 62 for testing the performance of the predictors. A preliminary analysis revealed that the signals input to MAPPP $P_{\mathrm{RAD}}$ are not sufficient for classification. A success rate no higher than $60 \%$ is very difficult to achieve. This is due to the fact that these signals cannot identify the radiative disruptions. As additional inputs, the ratio of various bolometric chords have been selected. The bolometric diagnostic is available routinely an in real time on JET and therefore this is a choice 
compatible with real time applications. The layout of the diagnostic is reported in Figure 14. The chords analysed are 24 of the vertical and 24 of the horizontal camera.

The tool implemented to select the most relevant chords to perform the classification is the Classification And Regression Trees (CART) approach. CART is a machine learning technique, which constructs a model starting from the data [24]. The objective consists of classifying a target variable based on the information provided by a series of observations.

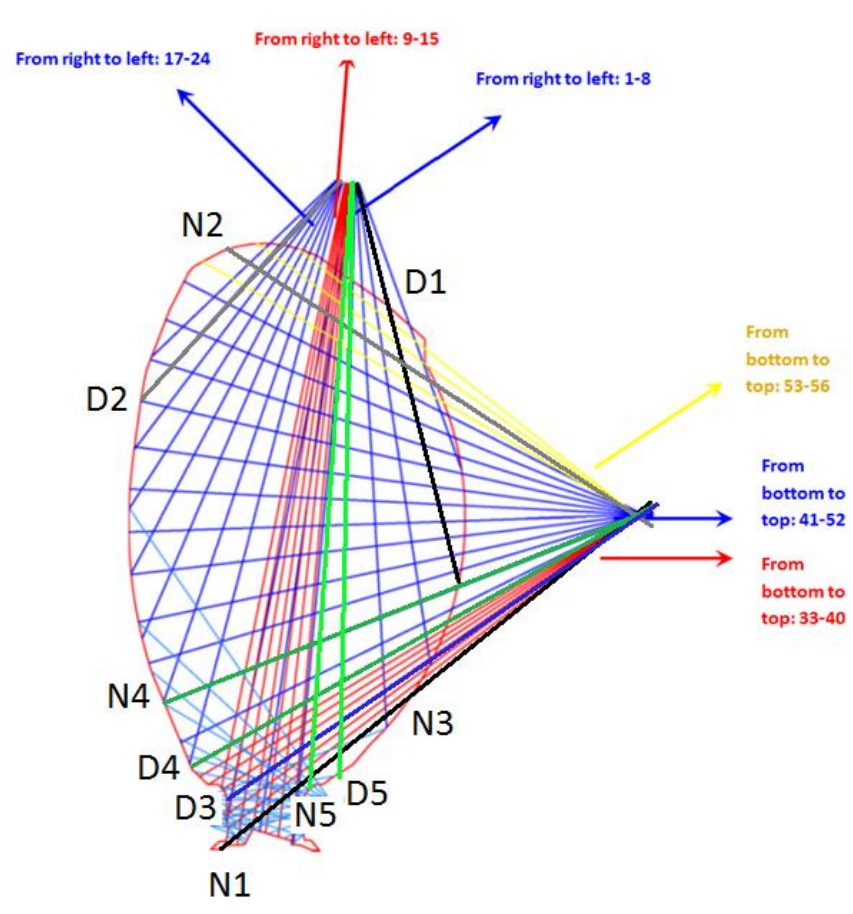

Figure 14. Layout of JET bolometric cameras. $N_{i}$ and $D_{i}$ indicate the chords used respectively as numerators and denominators in the five ratios provided as inputs to MAPP ${ }_{\text {Class. }}$
The model output is a tree, whose internal nodes, also called non-leaf nodes, represent conditions on the observations. Each leaf of the tree is labelled with a class. The arc of the tree from the root to a leaf identifies a series of conditions, which when satisfied allow attributing to a new example the label of the leaf. The CART training, the algorithm that determines the classification tree on the basis of the set of examples, consists of recursively partitioning the data and finding the partition which minimizes the impurity of the leaf nodes. The impurity of a node is calculated with the Gini coefficient. Since CART does not provide probabilities, in our application this machine learning approach has been used for the selection of the most relevant chord ratios. Since it is well known that trees ae no very robust classifiers, the CART routines have been runs tens of times and the chord ratios most commonly identified have been selected for as inputs to a version of the SVM-based probabilistic classifier called MAPP ${ }_{\text {Class. }}$ The five ratios finally selected are:

1. Ratio 1: Horizontal chord \#1

2. Ratio 2: Horizontal chord \#22

3. Ratio 3: Horizontal chord \#1

4. Ratio 4: Horizontal chord \#10

5. Ratio 5: Vertical chord \#2
/ Vertical chord \#2

/ Vertical chord \#23

/ Horizontal chord \#4

/ Horizontal chord \#8

/ Vertical chord \#8 
These five ratios of bolometric channels have been used as inputs to MAPP $\mathrm{Class}$ together with the internal inductance and the radiated fraction (basically the chord ratios have been added to the inputs of MAPPPRAD).

The success rate of the classifier is reported in Table IV, for a threshold in probability of $80 \%$. The performance of $\mathrm{MAPP}_{\text {Class }}$ are certainly not completely satisfactory. On the other

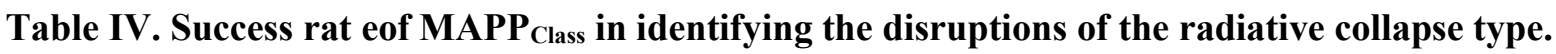

\begin{tabular}{|c|c|c|}
\hline $\begin{array}{c}\text { Total Success Rate } \\
{[\%]}\end{array}$ & $\begin{array}{c}\text { Success Rate Radiation Limit Class } \\
{[\%] \text { and ratio }}\end{array}$ & $\begin{array}{c}\text { Success Rate Others } \\
{[\%] \text { and ratio }}\end{array}$ \\
\hline 77.4 & $80 \quad(8 / 10)$ & $76.9 \quad(40 / 52)$ \\
\hline
\end{tabular}

hand, various extenuating circumstances should be considered. First of all the complexity of the classification task, which renders the set of examples available very limited. Second, the nature of the classification itself; from a manual inspection of the data it appears very clearly that the two classes, radiative and other, are not very well separated. There are other types of disruptions, which are characterised by a very high radiated fraction. Therefore, the two classes are not completely mutually exclusive and this explains in particular the relatively high rate of errors in classifying the disruptions in the class others. On the other hand, this example is sufficient to support statement that the technology developed is more than adequate also for classification and hat much better results can be expected once various aspects of the database and the input signals are improved.

\section{Discussion and Conclusions}

The development of an SVM-based probabilistic classifier has allowed the implementation of a series of tools for disruption prediction, which provide very competitive performance. The generality of the developed tools has been confirmed by their deployment to predict disruptions in JET for both the Carbon wall and the ITER Like Wall. These tools are also very flexible and allow attacking all the main issues related to disruption forecasting in Tokamaks, from prediction for mitigation to avoidance and classification. Of course, the quality of the final results, particularly for the classification of the disrupting types, is predicated on the identification of adequate signals with sufficient information content. In any case, the output of such tools being a probability is a fact that increases their interpretability. Moreover, probabilistic predictors are certainly more suited to the integration 
into complex decision-making systems than simple binary classifiers, which do not qualify the quality of their results.

As mentioned one of the main advantages of the probabilistic predictors of the MAPP family resides certainly in the flexibility provided by their probabilistic output. This information allows optimising the predictors and fine-tuning them for any specific application. On the other hand, the MAPP family remains a data driven approach to prediction. Therefore, the performance of these tools are ultimately determined by the information content of the signals used as inputs. This has been clearly shown by the analysis of the temporal evolution of the probability of disruption in the case of the locked mode and the radiated fraction as inputs. The limits of the statistics available and the signals are particularly evident for the more complex task of classification.

In terms of practical applications, in the short term it is planned to use the developed tools for selecting the most appropriate threshold for the LMPT predictor, the one routinely used on JET for triggering mitigation actions. On the other hand, the main line of future investigations is expected to be the adaptation of the developed tools for avoidance. This is a quite innovative field for machine learning predictors, which have been so far mainly used for mitigation. To extend the applicability and improve the performance for avoidance, first a careful selection of the input signals will have to be performed. As shown in Section 6, analysing the radiated power seems to be a very promising alternative. Probably more sophisticated indicator than the simple total radiated fraction will have to be provided as input to the predictors in order to improve significantly the results. The peaking of the radiation, or in any case information about the radiation profile, are probably quite good candidates. Other profiles, such as the one of the temperature, will also have to be seriously considered.

In addition to a proper selection of additional quantities, the training procedures will likely have to be refined. More sophisticated approaches will have to be developed to identify appropriate precursors and to use their information in a reliable way. More complex logic would probably also be necessary. As an example, to better profit from the effects of impurity on radiation, it is possible that different predictors will have to be trained for different levels of input power. Indeed, it is not obvious that a certain device can operate safely at the same radiated fraction at all levels of input power.

With regard to other future applications, the same tools described in this paper can be profitably used to investigate other aspects of Tokamak physics. A natural potential extension of the methodology could be the investigation of the $\mathrm{L}-\mathrm{H}$ transition physics, to complement the approach already presented in [25]. This problem can indeed also be formulated as a 
boundary between different operational regimes [26] and could be therefore analysed with the tools developed for the present work.

\begin{abstract}
Aknowledgements
This work has been carried out within the framework of the EUROfusion Consortium and has received funding from the Euratom research and training programme 2014-2018 under grant agreement No 633053. The views and opinions expressed herein do not necessarily reflect those of the European Commission. This work was also partially supported by the Spanish Ministry of Economy and Competitiveness under the Projects Nos. ENE2015-64914-C3-1-R and ENE2015-64914-C3-2-R.
\end{abstract}

\title{
References
}

[1] C.R.Hadlock "Six causes of Collapse" Mathematical Association of America Washington 2012

[2] R.Wenninger et al "Power Handling and Plasma Protection Aspects that affect the Design of the DEMO Divertor and First Wall' submitted for publication in Proceedings of 26th IAEA Fusion Energy Conference

[3] A.Murari et al Nucl. Fusion 57 (2017) 016024 (11pp) doi:10.1088/0029$5515 / 57 / 1 / 016024$

[4] P. Domingos "The Master Algorithm: How the Quest for the Ultimate Learning Machine Will Remake Our World" Basic Bools, 2015 NY

[5] A.Murari et al Nuclear Fusion, Volume 49, Number 5 April 2009 doi.org/10.1088/0029$5515 / 49 / 5 / 055028$

[6] A.Murari et al Nuclear Fusion, Volume 48, Number 3 February 2008 doi.org/10.1088/0029-5515/48/3/035010

[7] G.Rattà et al Nuclear Fusion, Volume 50, Number 2 January 2010 doi.org/10.1088/0029$5515 / 50 / 2 / 025005$

[8] Y.Zhang et al Nuclear Fusion, Volume 51, Number 6 May 2011 doi.org/10.1088/0029$5515 / 51 / 6 / 063039$

[9] J. Vega, S. Dormido-Canto, J. M. López, A. Murari, J. M. Ramírez, R. Moreno, M. Ruiz, D. Alves, R. Felton and JET-EFDA Contributors. "Results of the JET real-time disruption 
predictor in the ITER-like wall campaigns". Fusion Engineering and Design 88 (2013) 12281231.

[10] J. Vega, R. Moreno, A. Pereira, S. Dormido-Canto, A. Murari and JET Contributors. “Advanced disruption predictor based on the locked mode signal: application to JET". $1^{\text {st }}$ EPS Conference on Plasma Diagnostics. April 14-17, 2015. Book of abstracts. Frascati, Italy. [11] .Vega, A. Murari, S. Dormido-Canto, R. Moreno, A. Pereira, G. A. Rattá and JET Contributors. "Disruption Precursor Detection: Combining the Time and Frequency Domains". Proc. of the 26th Symposium on Fusion Engineering (SOFE 2015). May 31stJune 4th, 2015. Austin (TX), USA

[12] J. Vega, R. Moreno, A. Pereira, S. Dormido-Canto, A. Murari and JET Contributors. "Advanced disruption predictor based on the locked mode signal: application to JET". Proceedings of Science . ECPD 2015, 028

[13] J. Vega, A. Murari, S. Dormido-Canto, R. Moreno, A. Pereira, A. Acero and JET-EFDA Contributors. "Adaptive high learning rate probabilistic disruption predictors from scratch for the next generation of tokamaks". Nuclear Fusion. 54 (2014) 123001 (17pp).

[14] S. Dormido-Canto, J. Vega, J. M. Ramírez, A. Murari, R. Moreno, J. M. López, A. Pereira and JET-EFDA Contributors. "Development of an efficient real-time disruption predictor from scratch on JET and implications for ITER”. Nuclear Fusion. 53 (2013) 113001 (8pp).

[15] Steinwart, Ingo; and Christmann, Andreas; Support Vector Machines, Springer-Verlag, New York, 2008. ISBN 978-0-387-77241-7

[16] Platt, J. C. Probabilistic outputs for support vector machines and comparisons to regularized likelihood methods. In A. Smola et al. (ed.), Advances in Large Margin Classifiers. MIT Press, Cambridge, MA, 2000.

[17] C. Lin, C. Hsu. "A comparison of methods for multiclass support vector Machines".

IEEE Transactions on Neural Networks 13 (2002)

[18] J. Weston, C. Watkins, "Support Vector Machines for multi-class pattern recognition".

Proceeding of the Seventh European Symposium on Artificial Neural Networks, 1999

[19] J. M. López, J. Vega, D. Alves, S. Dormido-Canto, A. Murari, J. M. Ramírez, R. Felton, M. Ruiz, G. de Arcas and JET-EFDA Contributors. "Implementation of the Disruption Predictor APODIS in JET's Real-Time Network Using the MARTe Framework". IEEE Transactions on Nuclear Science. 61, 2 (2014), 741-744.

[20] P.C. de Vries et al Nuclear Fusion, Volume 56, Number 2 December 2015 doi.org/10.1088/0029-5515/56/2/026007 
[21] A. Murari et al Nuclear Fusion, Volume 57, Number 1 November 2016 doi.org/10.1088/0029-5515/57/1/016024

[22] A.Murari et al Nuclear Fusion, Volume 53, Number 3 February 2013 doi.org/10.1088/0029-5515/53/3/033006

[23] B.Cannas et al Nuclear Fusion, Volume 53, Number 9 August 2013 doi.org/10.1088/0029-5515/53/9/093023

[24] L. Breiman, J. H. Friedman, R. A. Olshen, C. J. Stone "Classification and Regression Trees" Chapman\&Hall, London 1998.

[25]J. Vega, A. Murari, G. Vagliasindi, G. A. Rattá and JET-EFDA Contributors.

"Automated estimation of $\mathrm{L} / \mathrm{H}$ transition times at JET by combining Bayesian statistics and Support Vector Machines”. Nuclear Fusion. 49 (2009) 085023 (11pp).

[26] P. Gaudio, A. Murari, M. Gelfusa, I. Lupelli, J. Vega) Plasma Physics and Controlled Fusion, iop publishing ltd, volume 56, 2014. 
APPENDIX A: Performance of MAPP for various choice of the triggering window: database of JET with the ILW.

Table A1. Main figures of merit of MAPP quality using the posterior probability to decide whether to trigger an alarm. This adaptive predicators have been implemented triggering an alarm after two consecutive time slices detect a disruption.

\begin{tabular}{|c|c|c|c|c|c|c|c|}
\hline $\begin{array}{c}\text { Thershold } \\
\text { post prob } \\
\text { DISR }\end{array}$ & $\begin{array}{c}\text { Succes Rate } \\
\%\end{array}$ & $\begin{array}{c}\text { Missed } \\
\%\end{array}$ & $\begin{array}{c}\text { Early } \\
\%\end{array}$ & $\begin{array}{c}\text { Tardy } \\
\%\end{array}$ & $\begin{array}{c}\text { False } \\
\%\end{array}$ & $\begin{array}{c}\text { Mean } \\
{[\mathbf{m s}]}\end{array}$ & $\begin{array}{c}\text { Std } \\
{[\mathrm{ms}]}\end{array}$ \\
\hline 30 & 91.40 & 0.54 & 5.37 & 2.69 & 11.70 & 314 & 327 \\
\hline 40 & 94.08 & 0.54 & 2.69 & 2.69 & 6.37 & 314 & 329 \\
\hline 50 & 95.16 & 0.54 & 2.15 & 2.15 & 5.79 & 317 & 332 \\
\hline 60 & 95.70 & 0.54 & 1.61 & 2.15 & 5.55 & 321 & 336 \\
\hline 70 & 95.70 & 0.54 & 1.61 & 2.15 & 5.40 & 321 & 335 \\
\hline 80 & 94.62 & 0.54 & 2.15 & 2.69 & 5.69 & 312 & 331 \\
\hline 90 & 96.23 & 0.54 & 0.54 & 2.69 & 4.03 & 301 & 326 \\
\hline
\end{tabular}

Table A2. Main figures of merit of MAPP quality using the posterior probability to decide whether to trigger an alarm. This adaptive predicators have been implemented triggering an alarm after three consecutive time slices detect a disruption.

\begin{tabular}{|c|c|c|c|c|c|c|c|}
\hline $\begin{array}{c}\text { Thershold } \\
\text { post prob } \\
\text { DISR }\end{array}$ & $\begin{array}{c}\text { Succes Rate } \\
\%\end{array}$ & $\begin{array}{c}\text { Missed } \\
\%\end{array}$ & $\begin{array}{c}\text { Early } \\
\%\end{array}$ & $\begin{array}{c}\text { Tardy } \\
\%\end{array}$ & $\begin{array}{c}\text { False } \\
\%\end{array}$ & $\begin{array}{c}\text { Mean } \\
{[\mathbf{m s}]}\end{array}$ & $\begin{array}{c}\text { Std } \\
{[\mathbf{m s}]}\end{array}$ \\
\hline 30 & 91.93 & 0.54 & 5.37 & 2.15 & 11.61 & 314 & 327 \\
\hline 40 & 94.08 & 0.54 & 3.22 & 2.15 & 6.27 & 314 & 329 \\
\hline 50 & 94.62 & 0.54 & 2.15 & 2.69 & 5.79 & 317 & 332 \\
\hline 60 & 96.24 & 0.54 & 1.07 & 2.15 & 5.40 & 321 & 335 \\
\hline 70 & 95.16 & 0.54 & 1.61 & 2.69 & 5.79 & 320 & 335 \\
\hline 80 & 95.70 & 0.54 & 0.54 & 3.22 & 4.43 & 312 & 331 \\
\hline 90 & 96.24 & 0.54 & 0.54 & 2.69 & 4.23 & 310 & 326 \\
\hline
\end{tabular}


APPENDIX B: Performance of the MAPP ${ }_{\text {Carbon }}$ for various choice of the triggering window: database of JET with the graphite wall.

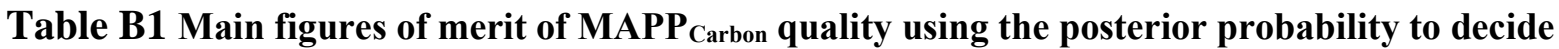
whether to trigger an alarm. This adaptive predicators have been implemented triggering an alarm after the first time slice detecting a disruption.

\begin{tabular}{|c|c|c|c|c|c|c|c|}
\hline $\begin{array}{c}\text { Threshold } \\
\text { post prob } \\
\text { DISR }\end{array}$ & $\begin{array}{c}\text { Succes } \\
\text { Rate }\end{array}$ & Missed & Early & Tardy & False & Mean & Std \\
\hline 30 & 91.55 & 1.41 & 4.93 & 2.11 & 6.14 & 264 & 321 \\
\hline 40 & 93.66 & 0.70 & 3.52 & 2.11 & 5.80 & 272 & 344 \\
\hline 50 & 93.66 & 0.00 & 3.52 & 2.81 & 4.32 & 264 & 333 \\
\hline 60 & 92.96 & 0.00 & 4.22 & 2.81 & 3.89 & 276 & 346 \\
\hline 70 & 95.07 & 0.00 & 2.11 & 2.81 & 3.41 & 277 & 349 \\
\hline 80 & 95.77 & 0.70 & 1.41 & 2.11 & 2.64 & 270 & 332 \\
\hline 90 & 95.77 & 0.70 & 1.41 & 2.11 & 2.64 & 257 & 314 \\
\hline
\end{tabular}

Table B2 Main figures of merit of MAPP ${ }_{\text {Carbon }}$ quality using the posterior probability to decide whether to trigger an alarm. This adaptive predicators have been implemented triggering an alarm after two consecutive time slices detecting a disruption.

\begin{tabular}{|c|c|c|c|c|c|c|c|}
\hline $\begin{array}{c}\text { Threshold } \\
\text { post prob } \\
\text { DISR }\end{array}$ & $\begin{array}{c}\text { Succes } \\
\text { Rate }\end{array}$ & Missed & Early & Tardy & False & Mean & Std \\
\hline 30 & 88.73 & 1.41 & 7.04 & 2.81 & 4.93 & 274 & 340 \\
\hline 40 & 89.44 & 1.41 & 6.34 & 2.81 & 4.36 & 287 & 368 \\
\hline 50 & 90.14 & 1.41 & 5.63 & 2.81 & 3.69 & 280 & 367 \\
\hline 60 & 92.25 & 2.81 & 2.81 & 2.11 & 3.60 & 264 & 318 \\
\hline 70 & 92.95 & 2.11 & 2.81 & 2.11 & 3.31 & 259 & 317 \\
\hline 80 & 93.66 & 2.11 & 2.11 & 2.11 & 2.73 & 260 & 313 \\
\hline 90 & 92.25 & 2.81 & 2.11 & 2.81 & 2.21 & 260 & 316 \\
\hline
\end{tabular}




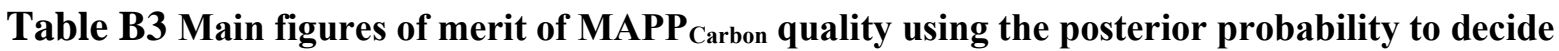
whether to trigger an alarm. This adaptive predicators have been implemented triggering an alarm after three consecutive time slices detect a disruption.

\begin{tabular}{|c|c|c|c|c|c|c|c|}
\hline $\begin{array}{c}\text { Threshold } \\
\text { post prob } \\
\text { DISR }\end{array}$ & $\begin{array}{c}\text { Succes } \\
\text { Rate }\end{array}$ & Missed & Early & Tardy & False & $\begin{array}{c}\text { Mean } \\
{[\mathbf{m s}]}\end{array}$ & $\begin{array}{c}\text { Std } \\
{[\mathbf{m s}]}\end{array}$ \\
\hline 30 & 87.32 & 2.11 & 7.74 & 2.81 & 5.40 & 266 & 340 \\
\hline 40 & 88.73 & 2.11 & 6.34 & 2.81 & 5.17 & 273 & 340 \\
\hline 50 & 89.44 & 2.11 & 5.63 & 2.81 & 4.84 & 286 & 371 \\
\hline 60 & 90.84 & 2.81 & 3.52 & 2.81 & 3.89 & 297 & 394 \\
\hline 70 & 91.55 & 2.11 & 2.81 & 3.52 & 3.17 & 257 & 316 \\
\hline 80 & 93.66 & 2.11 & 2.81 & 2.11 & 2.26 & 264 & 318 \\
\hline 90 & 93.66 & 2.11 & 0.70 & 3.52 & 2.21 & 269 & 334 \\
\hline
\end{tabular}

